\title{
Limits of level and parameter dependent subdivision schemes: A matrix approach
}

\author{
Maria Charina ${ }^{\mathrm{a}, *}$, Costanza Conti $^{\mathrm{b}}$, Nicola Guglielmi ${ }^{\mathrm{c}}$, Vladimir Protasov $^{\mathrm{d}}$ \\ a Fakultät für Mathematik, Universität Wien, Austria \\ ${ }^{\mathrm{b}}$ DIEF, University of Firenze, Italy

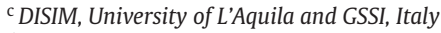 \\ ${ }^{\mathrm{d}}$ Moscow State University, Russia
}

\section{A R T I C L E I N F O}

\section{Article history:}

Available online 1 October 2015

\section{Keywords:}

Level dependent (non-stationary) subdivision

schemes

Tension parameter

Sum rules

Hölder regularity

Joint spectral radius

\begin{abstract}
A B S T R A C T
In this paper, we present a new matrix approach for the analysis of subdivision schemes whose non-stationarity is due to linear dependency on parameters whose values vary in a compact set. Indeed, we show how to check the convergence in $C^{\ell}\left(\mathbb{R}^{s}\right)$ and determine the Hölder regularity of such level and parameter dependent schemes efficiently via the joint spectral radius approach. The efficiency of this method and the important role of the parameter dependency are demonstrated on several examples of subdivision schemes whose properties improve the properties of the corresponding stationary schemes.
\end{abstract}

(c) 2015 Elsevier Inc. All rights reserved.

\section{Introduction}

We analyze convergence and Hölder regularity of multivariate level dependent (non-stationary) subdivision schemes whose masks depend linearly on one or several parameters. For this type of schemes, which include well-known schemes with tension parameters [1,12,14,23,24,32], the theoretical results from [7] are applicable, but not always efficient. Indeed, if the level dependent parameters vary in some compact set, then the set of the so-called limit points (see [7]) of the corresponding sequence of non-stationary masks exists, but cannot be determined explicitly. This hinders the regularity analysis of such schemes. Thus, we present a different perspective on the results in [7] and derive a new general method for convergence and regularity analysis of such level and parameter dependent schemes. The practical efficiency of this new method is illustrated in several examples.

Subdivision schemes are iterative algorithms for generating curves and surfaces from given control points of a mesh. They are easy to implement and intuitive in use. These and other nice mathematical properties of subdivision schemes motivate their popularity in applications, i.e. in modeling of freeform curves and surfaces, approximation and interpolation of functions, computer animation, signal and image processing etc. Non-stationary subdivision schemes extend the variety of different shapes generated by stationary subdivision. Indeed, the level dependency enables to generate new classes of functions such as exponential polynomials, exponential B-splines, etc. This gives a new impulse to development of subdivision schemes and enlarges the scope of their applications, e.g. in biological imaging [17,36], geometric design [34,37] or isogeometric analysis [2,9].

The main challenges in the analysis of any subdivision scheme are its convergence (in various function spaces), the regularity of its limit functions and its generation and reproduction properties. The important role of the matrix approach for regularity

\footnotetext{
* Corresponding author. Tel.: +43 1427755756 .

E-mail addresses: maria.charina@univie.ac.at (M. Charina), costanza.conti@unifi.it (C. Conti), guglielm@units.it (N. Guglielmi), v-protassov@yandex.ru (V. Protasov).
} 
analysis of stationary subdivision schemes is well-known. It allows to reduces the analysis to the computation or to the estimation of the joint spectral radius of the finite set of square matrices derived from the subdivision mask. Recent advances in the joint spectral radius computation [26,33] make the matrix approach very precise and efficient. In the non-stationary setting, however, this approach has never been applied because of the several natural obstacles. First of all, the matrix products that emerge in the study of non-stationary schemes have a different form than those usually analyzed by the joint spectral radius techniques. Secondly, the masks of non-stationary schemes do not necessarily satisfy sum rules, which destroys the relation between the convergence of the scheme and spectral properties of its transition matrices. All those difficulties were put aside by the results in [7], where the matrix approach was extended to the non-stationary setting.

In this paper, in Section 3, we make the next step and consider level and parameter dependent subdivision schemes whose masks include tension parameters, used to control the properties of the subdivision limit. Mostly, the tension parameters are level dependent and influence the asymptotic behavior of the scheme. If this is the case, the scheme can be analyzed by Charina et al. [7], which states that the convergence and Hölder regularity of any such non-stationary scheme depends on the joint spectral radius of the matrices generated by the so-called limit points of the sequence of level-dependent masks. In Theorem 3.5, we show that for the schemes with linear dependence on these parameters, the result of [7] can be simplified and be made more practical, see examples in Section 3.1.

\section{Background}

Let $m I \in \mathbb{Z}^{S \times s},|m| \geq 2$, be a dilation matrix and $E=\{0, \ldots,|m|-1\}^{s}$ be the set of the coset representatives of $\mathbb{Z}^{S} / m \mathbb{Z}^{S}$. We study subdivision schemes given by the sequence $\left\{S_{\mathbf{a}(r)}, r \in \mathbb{N}\right\}$ of subdivision operators $S_{\mathbf{a}(r)}: \ell\left(\mathbb{Z}^{S}\right) \rightarrow \ell\left(\mathbb{Z}^{S}\right)$ that define the subdivision rules by

$$
\left(S_{\mathbf{a}}^{(r)} \mathbf{c}\right)_{\alpha}=\sum_{\beta \in \mathbb{Z}^{s}} \mathrm{a}_{\alpha-m \beta}^{(r)} c_{\beta}, \quad \alpha \in \mathbb{Z}^{s}
$$

The masks $\mathbf{a}^{(r)}=\left\{\mathrm{a}_{\alpha}^{(r)} \in \mathbb{R}, \alpha \in \mathbb{Z}^{s}\right\}, r \in \mathbb{N}$, are sequences of real numbers and are assumed to be all supported in $\{0, \ldots, N\}^{s}$, $N \in \mathbb{N}$. For the set

$$
K=\sum_{r=1}^{\infty} m^{-r} G, \quad G=\{-|m|, \ldots, N+1\}^{s},
$$

the masks define the square matrices

$$
A_{\varepsilon, \mathrm{a}^{(r)}}^{(r)}=\left(\mathrm{a}_{m \alpha+\varepsilon-\beta}^{(r)}\right)_{\alpha, \beta \in K}, \quad r \in \mathbb{N}, \quad \varepsilon \in E .
$$

We assume that the level dependent symbols

$$
a^{(r)}(z)=\sum_{\alpha \in \mathbb{Z}^{s}} \mathrm{a}_{\alpha}^{(r)} z^{\alpha}, \quad z^{\alpha}=z_{1}^{\alpha_{1}} \cdot \ldots \cdot z_{s}^{\alpha_{s}}, \quad z \in(\mathbb{C} \backslash\{0\})^{s},
$$

of the subdivision scheme

$$
c^{(r+1)}=S_{\mathbf{a}^{(r)}} c^{(r)}=S_{\mathbf{a}^{(r)}} S_{\mathbf{a}^{(r-1)}} \ldots S_{\mathbf{a}^{(1)}} c^{(1)}, \quad r \in \mathbb{N}, \quad c^{(1)} \in \ell\left(\mathbb{Z}^{S}\right),
$$

satisfy sum rules of order $\ell+1, \ell \in \mathbb{N}_{0}$.

Definition 2.1. Let $\ell \in \mathbb{N}_{0}, r \in \mathbb{N}$. The symbol $a^{(r)}(z), z \in(\mathbb{C} \backslash\{0\})^{s}$, satisfies sum rules of order $\ell+1$ if

$$
a^{(r)}(1, \ldots, 1)=|m|^{s} \text { and } \max _{|\eta| \leq \ell} \max _{\epsilon \in \Xi \backslash\{1\}}\left|D^{\eta} a^{(r)}(\epsilon)\right|=0 \text {, }
$$

where $\Xi=\left\{e^{-i \frac{2 \pi}{|m|} \varepsilon}=\left(e^{-i \frac{2 \pi}{|m|} \varepsilon_{1}}, \ldots, e^{-i \frac{2 \pi}{|m|} \varepsilon_{s}}\right), \varepsilon \in E\right\}$ and $D^{\eta}=\frac{\partial^{\eta_{1}}}{\partial z_{1}^{\eta_{1}}} \ldots \frac{\partial \eta_{s}}{\partial z_{s}^{\eta_{s}}}$.

For more details on sum rules see e.g $[3,4,30]$.

Remark 2.2. (i) The assumption that all symbols $a^{(r)}(z)$ satisfy sum rules of order $\ell+1$, guarantees that the matrices $A_{\varepsilon, \mathrm{a}^{(r)}}^{(r)}, \varepsilon \in$ $E, r \in \mathbb{N}$, in (2.2) have common left-eigenvectors of the form

$$
(p(\alpha))_{\alpha \in K}, \quad p \in \Pi_{\ell}
$$

where $\Pi_{\ell}$ is the space of polynomials of degree less than or equal to $\ell$. Thus, the matrices $A_{\varepsilon, \mathbf{a}^{(r)}}^{(r)}, \varepsilon \in E, r \in \mathbb{N}$, possess a common linear subspace $V_{\ell} \subset \mathbb{R}^{|K|}$ orthogonal to the span of the common left-eigenvectors of $A_{\varepsilon, \mathrm{a}^{(r)}}^{(r)}, \varepsilon \in E, r \in \mathbb{N}$. The spectral properties of the set

$$
\mathcal{T}=\left\{\left.A_{\varepsilon, \mathbf{a}^{(r)}}^{(r)}\right|_{V_{\ell}}, \varepsilon \in E, r \in \mathbb{N}\right\}
$$

determine the regularity of the non-stationary scheme, see [7]. 
(ii) In the univariate case, the assumption that the symbols $a^{(r)}(z), r \in \mathbb{N}$, satisfy sum rules of order $\ell+1$ implies that

$$
a^{(r)}(z)=\left(1+z+\cdots+z^{|m|-1}\right)^{\ell} \sum_{\alpha \in \mathbb{Z}} b_{\alpha}^{(r)} z^{\alpha}, \quad z \in \mathbb{C} \backslash\{0\},
$$

and

$$
\left.A_{\varepsilon, \mathrm{a}^{(r)}}^{(r)}\right|_{V_{\ell}}=\left(b_{m \alpha+\varepsilon-\beta}^{(r)}\right)_{\alpha, \beta \in\{0, \ldots, N-\ell\}}, \quad \varepsilon \in E .
$$

In the multivariate case, the explicit form of the matrices $\left.A_{\varepsilon, \mathrm{a}}^{(r)}\right|_{V_{\ell}}, \varepsilon \in E, r \in \mathbb{N}$, depends on the choice of the basis of $V_{\ell}$, see e.g. [3] or [7].

Before stating the definition of the $C^{\ell}$-convergence of a subdivision scheme we recall the notion of a test function from [15].

Definition 2.3. A scalar valued function $f \in L_{\infty}\left(\mathbb{R}^{s}\right)$ is called a test function, if

(i) $f$ is compactly supported,

(ii) there exist constants $0<C_{1} \leq C_{2}<\infty$ such that

$$
C_{1} \sup _{\alpha \in \mathbb{Z}^{s}}\left|c_{\alpha}\right| \leq \sup _{x \in \mathbb{R}^{s}}\left|\sum_{\alpha \in \mathbb{Z}^{s}} c_{\alpha} f(x-\alpha)\right| \leq C_{2} \sup _{\alpha \in \mathbb{Z}^{s}}\left|c_{\alpha}\right|, \quad c \in \ell_{\infty}\left(\mathbb{Z}^{s}\right),
$$

(iii) the Fourier transform $\hat{f}(y)=\int_{\mathbb{R}^{s}} f(x) e^{-2 \pi i x \cdot y} d x$ of $f$ satisfies

$$
\hat{f}(0)=1, \quad D^{\mu} \hat{f}(\alpha)=0, \quad \alpha \in \mathbb{Z}^{S} \backslash\{0\}, \quad \mu \in \mathbb{N}_{0}^{S}, \quad|\mu|<\ell+1 .
$$

For more details on test functions see [15].

Definition 2.4. A subdivision scheme $\left\{S_{\mathbf{a}(r)}, r \in \mathbb{N}\right\}$ is $C^{\ell}$-convergent, if for any initial sequence $\mathbf{c} \in \ell_{\infty}\left(\mathbb{Z}^{s}\right)$ there exists a limit function $g_{c} \in C^{\ell}\left(\mathbb{R}^{S}\right)$ such that for any test function $f \in C^{\ell}\left(\mathbb{R}^{S}\right)$

$$
\lim _{k \rightarrow \infty}\left\|g_{\mathbf{c}}(\cdot)-\sum_{\alpha \in \mathbb{Z}^{S}} S_{\mathbf{a}^{(r)}} S_{\mathbf{a}^{(r-1)}} \ldots S_{\mathbf{a}^{(1)}} c_{\alpha} f\left(m^{k} \cdot-\alpha\right)\right\|_{C^{\ell}}=0 .
$$

Note that, it suffices to check (2.5) for only one test function $f$. Note also that, if all limits of a subdivision scheme belong to $C^{\ell}\left(\mathbb{R}^{s}\right)$, then the scheme may not converge in $C^{\ell}$, but only in $C^{0}\left(\mathbb{R}^{s}\right)$.

In this paper, we also show how to estimate the Hölder regularity of subdivision limits.

Definition 2.5. The Hölder regularity of the $C^{0}$-convergent scheme $\left\{S_{\mathbf{a}^{(r)}}, r \in \mathbb{N}\right\}$ is $\alpha=\ell+\zeta$, if $\ell$ is the largest integer such that $g_{\mathbf{c}} \in C^{\ell}\left(\mathbb{R}^{S}\right)$ and $\zeta$ is the supremum of $v \in[0,1]$ such that

$$
\max _{\mu \in \mathbb{N}_{0}^{s},|\mu|=\ell}\left|D^{\mu} g_{\mathbf{c}}(x)-D^{\mu} g_{\mathbf{c}}(y)\right| \leq|x-y|^{\nu}, \quad x, y \in \mathbb{R}^{s} .
$$

We call $\alpha$ the Hölder exponent of $\left\{S_{\mathbf{a}^{(r)}}, r \in \mathbb{N}\right\}$.

The joint spectral radius of a set of square matrices was introduced in [35].

Definition 2.6. The joint spectral radius (JSR) of a compact family $\mathcal{M}$ of square matrices is defined by

$$
\rho(\mathcal{M}):=\lim _{n \rightarrow \infty} \max _{M_{1}, \ldots, M_{n} \in \mathcal{M}}\left\|\prod_{j=1}^{n} M_{j}\right\|^{1 / n} .
$$

Note that the definition of the JSR is independent of the choice of the matrix norm $\|\cdot\|$.

The link between the JSR and stationary subdivision is well-known, see e.g. [5,8,16,29,31]. The results of [7] establish a link between the regularity of non-stationary subdivision schemes and the JSR of compact matrix sets derived from the limit points of $\left\{\mathbf{a}^{(r)}, r \in \mathbb{N}\right\}$.

Definition 2.7. For the mask sequence $\left\{\mathbf{a}^{(r)}, r \in \mathbb{N}\right\}$ we denote by $\mathcal{A}$ the set of its limit points, i.e. the set of masks a such that

$$
\mathbf{a} \in \mathcal{A}, \quad \text { if } \exists\left\{r_{n}, n \in \mathbb{N}\right\} \text { such that } \lim _{n \rightarrow \infty} \sup _{\alpha \in \mathbb{Z}^{s}}\left|\mathrm{a}_{\alpha}^{\left(r_{n}\right)}-\mathrm{a}_{\alpha}\right|=0 \text {. }
$$

For the readers convenience, we recall here the statement of [7] which is crucial for the proof of our main result, see Theorem 3.4.

Corollary 2.8. Let $\ell \geq 0$. Assume that the symbols of the scheme $\left\{S_{\mathbf{a}(r)}, r \in \mathbb{N}\right\}$ satisfy sum rules of order $\ell+1$ and $\rho_{\mathcal{A}}:=\rho\left(\mathcal{T}_{\mathcal{A}}\right)<$ $|m|^{-\ell}$, where

$$
\mathcal{T}_{\mathcal{A}}=\left\{\left.A_{\varepsilon, \mathbf{a}}\right|_{V_{\ell}}, \varepsilon \in E, \mathbf{a} \in \mathcal{A}\right\}
$$

and $\mathcal{A}$ is the set of the limit points of $\left\{\mathbf{a}^{(r)}, r \in \mathbb{N}\right\}$. Then $\left\{S_{\mathbf{a}}(r), r \in \mathbb{N}\right\}$ is $C^{\ell}$-convergent and the Hölder exponent of its limit functions is $\alpha \geq-\log _{|m|} \rho_{\mathcal{A}}$. 


\section{Parameter dependent subdivision schemes: matrix approach}

There are several examples of subdivision schemes that include a tension parameter. We call them parameter dependent schemes. Often, the tension parameter is level dependent and shows a certain asymptotic behavior which implies the asymptotic behavior of the corresponding non-stationary scheme, i.e. $\lim _{r \rightarrow \infty} \sup _{\alpha \in \mathbb{Z}^{s}}\left|\mathrm{a}_{\alpha}^{(r)}-\mathrm{a}_{\alpha}\right|=0$. In this case, although the set $\left\{A_{\varepsilon, \mathrm{a}^{(r)}}^{(r)}, \varepsilon \in\right.$ $E, r \in \mathbb{N}\}$ is not compact, the convergence and regularity of the scheme $\left\{S_{\mathbf{a}^{(r)}}, r \in \mathbb{N}\right\}$ can be analyzed via the joint spectral radius approach in [7]. The results in [7] are still applicable even if the parameter values vary in some compact interval. Indeed, in this case, the existence of the limit points for the sequence $\left\{\mathbf{a}^{(r)}, r \in \mathbb{N}\right\}$ of the subdivision masks is guaranteed, though these limit points are not always explicitly known. In this section, we show that the joint spectral radius approach can be effectively applied even if the limit points of $\left\{\mathbf{a}^{(r)}, r \in \mathbb{N}\right\}$ cannot be determined explicitly, but the masks $\mathbf{a}^{(r)}$ depend linearly on the parameter $\omega^{(r)}$ $\in\left[\omega_{1}, \omega_{2}\right],-\infty<\omega_{1}<\omega_{2}<\infty$.

A well-known and celebrated example of the parameter dependent stationary subdivision scheme with linear dependence on the parameter is the univariate four point scheme [19] with the symbol

$$
a(z, \omega)=z^{-1} \frac{(1+z)^{2}}{2}+\omega\left(-z^{-3}+z^{-1}+z-z^{3}\right), \quad \omega \in\left[0, \frac{1}{8}\right], \quad z \in \mathbb{C} \backslash\{0\},
$$

which is a parameter perturbation of the linear B-spline. Also the bivariate butterfly scheme [21] with the symbol

$$
a\left(z_{1}, z_{2}, \omega\right)=\frac{1}{2}\left(1+z_{1}\right)\left(1+z_{2}\right)\left(1+z_{1} z_{2}\right)+\omega c\left(z_{1}, z_{2}\right), \quad z_{1}, z_{2} \in \mathbb{C} \backslash\{0\},
$$

with

$$
\begin{aligned}
c\left(z_{1}, z_{2}\right)= & z_{1}^{-1} z_{2}^{-2}+z_{2}^{2} z_{1}^{-1}+z_{1}^{-2} z_{2}^{-1}+z_{1}^{2} z_{2}^{-1}-2 z_{1}^{2} z_{2}^{3}-2 z_{1}^{3} z_{2}^{2}+z_{1}^{2} z_{2}^{4}+z_{1}^{4} z_{2}^{2}+z_{1}^{3} z_{2}^{4} \\
& +z_{1}^{4} z_{2}^{3}-2 z_{1}^{-1}+z_{1}^{-2}-2 z_{1}^{2}-2 z_{2}^{-1}+z_{1}^{3}+z_{2}^{-2}-2 z_{2}^{2}+z_{2}^{3},
\end{aligned}
$$

is a parameter perturbation of the linear three-directional box spline. Other examples of such parameter dependent schemes are those with symbols that are convex combinations

$$
\omega a\left(z_{1}, z_{2}\right)+(1-\omega) b\left(z_{1}, z_{2}\right)=b\left(z_{1}, z_{2}\right)+\omega\left(a\left(z_{1}, z_{2}\right)-b\left(z_{1}, z_{2}\right)\right), \quad \omega \in[0,1], \quad z_{1}, z_{2} \in \mathbb{C} \backslash\{0\},
$$

of two (or more) symbols of stationary schemes, see e.g. [6,11,13]. Known are also (see e.g. [1,12,14]) their non-stationary univariate counterparts with the symbols

or

$$
z^{-1} \frac{(1+z)^{2}}{2}+\omega^{(r)}\left(-z^{-3}+z^{-1}+z-z^{3}\right), \quad r \in \mathbb{N}, \quad \lim _{r \rightarrow \infty} \omega^{(r)}=\omega \in \mathbb{R},
$$

$$
\omega^{(r)} a(z)+\left(1-\omega^{(r)}\right) b(z), \quad r \in \mathbb{N}, \quad \omega^{(r)} \in[0,1] .
$$

Note that the use of the level dependent parameters sometimes allows us to enhance the properties of the existing stationary schemes (e.g. with respect to their smoothness, size of their support or reproduction and generation properties [6,11,12,14]).

In all schemes considered above, the subdivision rules depend either on the same, fixed, parameter $\omega=\omega^{(r)} \in\left[\omega_{1}, \omega_{2}\right]$ independent of $r$, or the parameters $\omega^{(r)} \in\left[\omega_{1}, \omega_{2}\right]$ are chosen in a such a way that either $\lim _{r \rightarrow \infty} \omega^{(r)}=\omega \in\left[\omega_{1}, \omega_{2}\right]$ or the corresponding non-stationary scheme is asymptotically equivalent to some known stationary scheme. For the definition of the asymptotical equivalence see [20].

The results in [32] employ special Laurent polynomial technique and yield sufficient conditions for $C^{\ell}$-convergence of parameter dependent subdivision schemes whose rules are level and/or location $\alpha \in \mathbb{Z}^{s}$ dependent.

In this section, we provide a matrix method for analyzing the Hölder regularity of more general subdivision schemes: we consider the level dependent masks $\mathbf{a}\left(\omega^{(r)}\right)=\left\{\mathrm{a}_{\alpha}\left(\omega^{(r)}\right), \alpha \in \mathbb{Z}^{S}\right\}, r \in \mathbb{N}$, and require that $\omega^{(r)} \in\left[\omega_{1}, \omega_{2}\right]$ without any further assumptions on the behavior of the sequence $\left\{\omega^{(r)}, r \in \mathbb{N}\right\}$. We assume, however, that each of the masks depends linearly on the corresponding parameter $\omega^{(r)}$.

The level dependent masks $\left\{\mathbf{a}\left(\omega^{(r)}\right), r \in \mathbb{N}\right\}$ define the corresponding square matrices which we denote by

$$
A_{\varepsilon, \omega^{(r)}}=\left(\mathrm{a}_{m \alpha+\varepsilon-\beta}\left(\omega^{(r)}\right)\right)_{\alpha, \beta \in K}, \quad \varepsilon \in E,
$$

and the level dependent symbols

$$
a\left(z, \omega^{(r)}\right)=\sum_{\alpha \in \mathbb{Z}^{s}} \mathrm{a}_{\alpha}\left(\omega^{(r)}\right) z^{\alpha}, \quad z^{\alpha}=z_{1}^{\alpha_{1}} \cdot \ldots \cdot z_{s}^{\alpha_{s}}, \quad z \in(\mathbb{C} \backslash\{0\})^{s} .
$$

The assumption that each mask $\mathbf{a}\left(\omega^{(r)}\right)$ depends linearly on $\omega^{(r)}$, leads to the following immediate, but crucial result.

Proposition 3.1. Let $\ell \in \mathbb{N}_{0}$ and $-\infty<\omega_{1}<\omega_{2}<\infty$. If every symbol of the sequence $\left\{a\left(z, \omega^{(r)}\right), r \in \mathbb{N}\right\}$ depends linearly on the parameter $\omega^{(r)} \in\left[\omega_{1}, \omega_{2}\right]$ and satisfies sum rules of order $\ell+1$, then every matrix in $\mathcal{T}=\left\{\left.A_{\varepsilon, \omega^{(r)}}\right|_{V_{\ell}}, \omega^{(r)} \in\left[\omega_{1}, \omega_{2}\right], \varepsilon \in E, r \in \mathbb{N}\right\}$ is a convex combination of the matrices with $\omega^{(r)} \in\left\{\omega_{1}, \omega_{2}\right\}$

$$
\left.A_{\varepsilon, \omega^{(r)}}\right|_{V_{\ell}}=\left.\left(1-t^{(r)}\right) A_{\varepsilon, \omega_{1}}\right|_{V_{\ell}}+\left.t^{(r)} A_{\varepsilon, \omega_{2}}\right|_{V_{\ell}}, \quad t^{(r)} \in[0,1]
$$


Proof. Let $r \in \mathbb{N}$. We first write $\omega^{(r)}$ as a convex combination of $\omega_{1}$ and $\omega_{2}$, i.e.

$$
\omega^{(r)}=\left(1-t^{(r)}\right) \omega_{1}+t^{(r)} \omega_{2} \quad \text { with } t^{(r)} \in[0,1] .
$$

Note that all entries of the matrices $A_{\varepsilon, \omega^{(r)}}, \varepsilon \in E$, are the coefficients of the corresponding mask a( $\left.\omega^{(r)}\right)$. Since the mask coefficients depend linearly on the parameter $\omega^{(r)}$, so do the matrices $A_{\varepsilon, \omega^{(r)}}$, and hence, the corresponding linear operators. Therefore, the restrictions of these operators to their common invariant subspace $V_{\ell}$ also depend linearly on these parameters.

In the level independent case, i.e. $\omega^{(r)}=\omega$ for all $r \in \mathbb{N}$, the use of the joint spectral radius approach for studying the convergence and regularity of the corresponding stationary subdivision schemes is well understood. To show how this approach can be applied in the non-stationary setting, we need first to prove the following auxiliary result.

Theorem 3.2. Let $\ell \in \mathbb{N}_{0}$ and

$$
\mathcal{T}=\left\{\left.A_{\varepsilon, \omega^{(r)}}\right|_{V_{\ell}}, \omega^{(r)} \in\left[\omega_{1}, \omega_{2}\right], \varepsilon \in E, r \in \mathbb{N}\right\}
$$

be the infinite family of square matrices. If the JSR of the family $\mathcal{T}_{\omega_{1}, \omega_{2}}=\left\{\left.A_{\varepsilon, \omega_{1}}\right|_{V_{\ell}},\left.A_{\varepsilon, \omega_{2}}\right|_{V_{\ell}}, \varepsilon \in E\right\}$ satisfies $\rho\left(\mathcal{T}_{\omega_{1}, \omega_{2}}\right)=\gamma$, then $\rho(\mathcal{T})=\gamma$.

Proof. Note that, for a compact set $\mathcal{M}$ of square matrices, an arbitrary vector norm $\|\cdot\|$ and $\delta>0$, the following norm

$$
\|x\|_{\delta}=\sup _{n \geq 0} \max _{M_{1}, \ldots, M_{n} \in \mathcal{M}} \frac{\left\|\prod_{j=1}^{n} M_{j} x\right\|}{(\rho(\mathcal{M})+\delta)^{n}}
$$

satisfies (see [28, (2.2), (2.3) and Proposition 2.2])

$$
\left\|\left.A_{\varepsilon, \omega_{1}}\right|_{V_{\ell}}\right\|_{\delta} \leq \gamma+\delta \text { and }\left\|\left.A_{\varepsilon, \omega_{2}}\right|_{V_{\ell}}\right\|_{\delta} \leq \gamma+\delta .
$$

This norm is called a $\delta$-extremal norm (see $[22,28]$ ).

Then, due to $\rho\left(\mathcal{T}_{\omega_{1}, \omega_{2}}\right)=\gamma$, by Proposition 3.1, by the estimates in (3.4) and by the subadditivity of matrix operator norms, we get

$$
\left\|\left.A_{\varepsilon, \omega^{(r)}}\right|_{V_{\ell}}\right\|_{\delta}=\left\|\left.\left(1-t^{(r)}\right) A_{\varepsilon, \omega_{1}}\right|_{V_{\ell}}+\left.t^{(r)} A_{\varepsilon, \omega_{2}}\right|_{V_{\ell}}\right\|_{\delta} \leq\left(1-t^{(r)}\right)\left\|\left.A_{\varepsilon, \omega_{1}}\right|_{V_{\ell}}\right\|_{\delta}+t^{(r)}\left\|\left.A_{\varepsilon, \omega_{2}}\right|_{V_{\ell}}\right\|_{\delta} \leq \gamma+\delta, \quad t^{(r)} \in[0,1] .
$$

This estimate, due to the arbitrary choice of $\delta>0$, implies that $\rho(\mathcal{T})=\gamma$, which concludes the proof.

Remark 3.3. $(i)$ Note that, if the family $\mathcal{T}_{\omega_{1}, \omega_{2}}$ is non-defective, i.e. there exists an extremal norm $\|\cdot\|$ such that $\max _{\varepsilon \in E}\left\{\left\|\left.A_{\varepsilon, \omega_{1}}\right|_{V_{\ell}}\right\|,\left\|\left.A_{\varepsilon, \omega_{2}}\right|_{V_{\ell}}\right\|\right\}=\gamma$, then $\mathcal{T}$ is also non-defective and all products of degree $d$ of the associated product semigroup have maximal growth bounded by $\gamma^{d}$. Note also that for any family of matrices $\mathcal{B}, \mathcal{B} \subset \mathcal{T}$, it follows that $\rho(\mathcal{B}) \leq \gamma$. (ii) It is not restrictive to assume that the family $\mathcal{T}_{\omega_{1}, \omega_{2}}$ is non-defective. Indeed, by Elsner [22], if the family $\mathcal{T}$ is irreducible (i.e. its matrices do not possess a common nontrivial subspace), then $\mathcal{T}$ is non-defective. And the irreducibility of $\mathcal{T}$ is the property that holds with probability 1 .

We are now ready to formulate the main result of this section.

Theorem 3.4. Let $\ell \in \mathbb{N}_{0}$. Assume that every symbol of the sequence $\left\{a\left(z, \omega^{(r)}\right), r \in \mathbb{N}\right\}$ depends linearly on $\omega^{(r)} \in\left[\omega_{1}\right.$, $\left.\omega_{2}\right]$ and satisfies sum rules of order $\ell+1$. Then the non-stationary scheme $\left\{S_{\mathbf{a}\left(\omega^{(r)}\right)}, r \in \mathbb{N}\right\}$ is $C^{\ell}$-convergent, if the JSR of the family $\mathcal{T}_{\omega_{1}, \omega_{2}}=$ $\left\{\left.A_{\varepsilon, \omega_{1}}\right|_{V_{\ell}},\left.A_{\varepsilon, \omega_{2}}\right|_{V_{\ell}}, \varepsilon \in E\right\}$ satisfies

$$
\gamma=\rho\left(\mathcal{T}_{\omega_{1}, \omega_{2}}\right)<|m|^{-\ell} \text {. }
$$

Moreover the Hölder exponent of its limit functions is $\alpha \geq-\log _{|m|} \gamma$.

Proof. Since the parameters $\left\{\omega^{(r)}, r \in \mathbb{N}\right\}$ vary in the compact interval $\left[\omega_{1}, \omega_{2}\right]$, there exists a set of limit points (finite or infinite) for the sequence $\left\{\mathbf{a}\left(\omega^{(r)}\right), r \in \mathbb{N}\right\}$ of subdivision masks. Let us denote this set by $\mathcal{A}$ and the corresponding set of square matrices by $\mathcal{T}_{\mathcal{A}}=\left\{\left.A_{\varepsilon}\right|_{V_{\ell}}=\left.\left(\mathrm{a}_{M \alpha+\varepsilon-\beta}\right)_{\alpha, \beta \in K}\right|_{V_{\ell}}, \varepsilon \in E, \mathbf{a} \in \mathcal{A}\right\}$. Obviously, $\mathcal{T}_{\mathcal{A}} \subset \mathcal{T}$ with $\mathcal{T}$ as in (3.3). Since by Theorem 3.2 and Remark 3.3 , $\rho\left(\mathcal{T}_{\mathcal{A}}\right) \leq \gamma$, the claim follows by Corollary 2.8 .

Remark 3.5. $(i)$ Note that, due to $\rho\left(\mathcal{T}_{\mathcal{A}}\right) \leq \gamma$, Theorem 3.4 yields a smaller Hölder exponent $\alpha$ than what could be obtained by Corollary 2.8. For example, consider a binary subdivision scheme with the symbols

$$
\begin{aligned}
& a\left(z, \omega^{(r)}\right)=z^{-1} \frac{(1+z)^{2}}{2}, \quad r \in\{1, \ldots, L\}, \\
& a\left(z, \omega^{(r)}\right)=z^{-1} \frac{(1+z)^{2}}{2}+\frac{1}{16}\left(-z^{-3}+z^{-1}+z-z^{3}\right), \quad r \geq L+1, \quad L \in \mathbb{N}, \quad z \in \mathbb{C} \backslash\{0\} .
\end{aligned}
$$


To apply Theorem 3.4, we can view the corresponding masks as being linearly dependent on parameters $\omega^{(r)} \in\left[0, \frac{1}{16}\right]$. The corresponding family $\mathcal{T}_{0, \frac{1}{16}}=\left\{\left.A_{\varepsilon, 0}\right|_{V_{1}},\left.A_{\varepsilon, \frac{1}{16}}\right|_{V_{1}}, \varepsilon \in\{0,1\}\right\}$ consists of the four matrices

$$
\left.A_{0, \omega}\right|_{V_{1}}=\left(\begin{array}{cccc}
-\omega & -2 \omega+\frac{1}{2} & -\omega & 0 \\
0 & 2 \omega & 2 \omega & 0 \\
0 & -\omega & -2 \omega+\frac{1}{2} & -\omega \\
0 & 0 & 2 \omega & 2 \omega
\end{array}\right),\left.\quad A_{1, \omega}\right|_{V_{1}}=\left(\begin{array}{cccc}
2 \omega & 2 \omega & 0 & 0 \\
-\omega & -2 \omega+\frac{1}{2} & -\omega & 0 \\
0 & 2 \omega & 2 \omega & 0 \\
0 & -\omega & -2 \omega+\frac{1}{2} & -\omega
\end{array}\right)
$$

for $\omega \in\left\{0, \frac{1}{16}\right\}$. Due to

$$
\max _{\varepsilon \in\{0,1\}}\left\{\left\|\left.A_{\varepsilon, 0}\right|_{V_{1}}\right\|_{\infty},\left\|\left.A_{\varepsilon, \frac{1}{16}}\right|_{V_{1}}\right\|_{\infty}\right\}=\max _{\varepsilon \in\{0,1\}}\left\{\rho\left(\left.A_{\varepsilon, 0}\right|_{V_{1}}\right), \rho\left(\left.A_{\varepsilon, \frac{1}{16}}\right|_{V_{1}}\right)\right\}=\frac{1}{2},
$$

we get $\rho\left(\mathcal{T}_{0, \frac{1}{16}}\right)=\frac{1}{2}$ and, thus, the corresponding scheme is convergent and has the Hölder exponent $\alpha \geq 1$. On the other hand, the set $\mathcal{A}$ of limit points of the masks can be explicitly determined in this case and consists of the mask of the four point scheme. Thus, by Corollary 2.8, the Hölder exponent is actually $\alpha \geq 2$.

(ii) The regularity estimate given in Theorem 3.4 can be improved, if the actual range of the parameters $\omega^{(r)}, r \geq L$, for some $L \in \mathbb{N}$, is a subinterval of $\left[\omega_{1}, \omega_{2}\right]$, see Section 3.1 .

(iii) Note that the result of Theorem 3.4 is directly extendable to the case when the matrix family $\mathcal{T}$ depends linearly on $\omega^{(r)}$, $r \in \mathbb{N}$, from the convex polyhedral set $\Omega=\overline{\operatorname{co}\left\{\omega_{1}, \ldots, \omega_{L}\right\}}$, the closed convex hull of $\omega_{1}, \ldots, \omega_{L} \in \mathbb{R}^{p}, L \in \mathbb{N}$, i.e.

$$
\omega^{(r)}=\sum_{j=1}^{L} t_{j}^{(r)} \omega_{j} \quad \text { with } t_{j}^{(r)} \in[0,1] \text { and } \sum_{j=1}^{L} t_{j}^{(r)}=1 .
$$

This is the case, for example, when we define the level and parameter dependent symbols

$$
a\left(z, \omega^{(r)}\right)=\sum_{j=1}^{p} \omega_{j}^{(r)} a_{j}(z), \quad \omega^{(r)}=\left(\omega_{1}^{(r)}, \ldots, \omega_{p}^{(r)}\right)^{T} \in \Omega, \quad r \in \mathbb{N} .
$$

\subsection{Examples}

In this section we present two univariate examples of level dependent parameter schemes, whose constructions are based on the four point and six point Dubuc-Deslauriers schemes. In particular, in Example 3.6, the non-stationary scheme is constructed in such a way that the support of its limit function

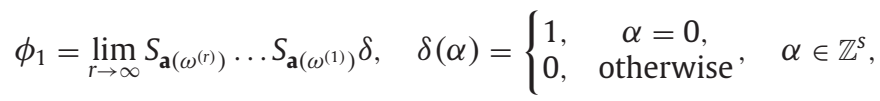

is smaller than the support of the four point scheme, but its regularity is comparable. In Example 3.7, every non-stationary mask is a convex combination of the four point and six point Dubuc-Deslauriers schemes. We show how the regularity of the corresponding non-stationary scheme depends on the range of the corresponding parameters $\left\{\omega^{(r)}, r \in \mathbb{N}\right\}$. Both examples illustrate the importance of the dependency on several parameters $\left\{\omega^{(r)}, r \in \mathbb{N}\right\}$ instead of one $\omega \in \mathbb{R}$.

We deliberately consider only univariate examples to keep the presentation easy to follow. Already in the bivariate case, the size of the matrices in the set (3.3) blows up. The techniques for their size reduction are known, but lead to intricate and lengthy matrix manipulations that we believe are out of scope of this paper.

Example 3.6. We consider the univariate, binary scheme with the symbols

$$
\begin{aligned}
& a\left(z, \omega^{(r)}\right)=z^{-1} \frac{(1+z)^{2}}{2}, \quad r \in\{1,2\}, \\
& a\left(z, \omega^{(r)}\right)=z^{-1} \frac{(1+z)^{2}}{2}+\omega^{(r)}\left(-z^{-3}+z^{-1}+z-z^{3}\right), \quad r \geq 3, \quad z \in \mathbb{C} \backslash\{0\},
\end{aligned}
$$

where $\omega^{(r)}$ are chosen at random from the interval $\left[\frac{3}{64}, \frac{1}{16}\right]$. The corresponding family

$$
\mathcal{T}_{0, \frac{1}{16}}=\left\{\left.A_{\varepsilon, 0}\right|_{V_{1}},\left.A_{\varepsilon, \frac{1}{16}}\right|_{V_{1}}, \varepsilon \in\{0,1\}\right\}
$$

consists of the same four matrices as in (3.6) and at the first glance the Hölder exponent of this scheme is $\alpha \geq 1$. On the other hand, we can view this scheme as the one with the corresponding matrix family

$$
\mathcal{T}_{\frac{3}{64}, \frac{1}{16}}=\left\{\left.A_{\varepsilon, \frac{3}{64}}\right|_{V_{1}},\left.A_{\varepsilon, \frac{1}{16}}\right|_{V_{1}}, \varepsilon \in\{0,1\}\right\},
$$

applied to a different starting data. (Recall that [[32], Theorem 3.1] shows that this scheme is $C^{1}$-convergent for $\omega^{(r)} \in\left[\epsilon, \frac{1}{8}-\epsilon\right]$, $r \geq 3,0<\epsilon<\frac{1}{16}$. ) We get $\rho\left(\mathcal{T}_{\frac{3}{64}, \frac{1}{16}}\right)=3 / 8$ and, by Theorem 3.4, the Hölder exponent is actually $\alpha \geq-\log _{2} \frac{3}{8} \approx 1.4150$. 


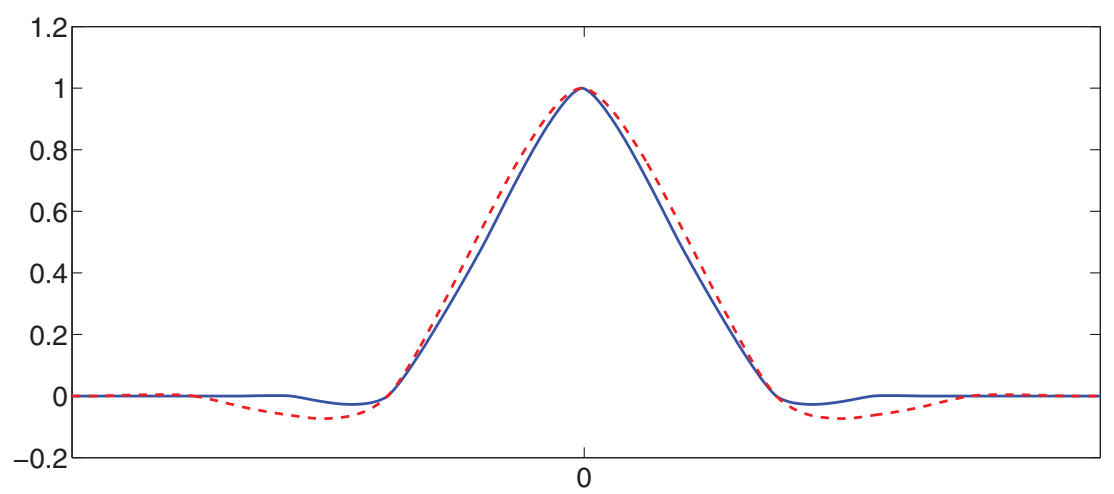

Fig. 1. Basic limit function of 4-point scheme (red, dashed line), limit function $\phi_{1}$ (blue, solid line). (For interpretation of the references to color in this figure legend, the reader is referred to the web version of this article.)

The size of the support of $\phi_{1}$ (see Fig. 1) can be determined using the technique from [10] and is given by

$$
\left[\sum_{r=0}^{\infty} 2^{-r-1} s(r), \sum_{r=0}^{\infty} 2^{-r-1} d(r)\right]=\left[-\frac{3}{2}, \frac{3}{2}\right]
$$

with

$$
\begin{array}{lll}
s(r)=-1, & d(r)=1, & r=0,1, \\
s(r)=-3, & d(r)=3, & r \geq 2 .
\end{array}
$$

Recall that the support of the basic limit function of the four point scheme is $[-3,3]$.

Example 3.7. In this example we consider the univariate non-stationary scheme with symbols

$$
a\left(z, \omega^{(r)}\right)=\omega^{(r)} a(z)+\left(1-\omega^{(r)}\right) b(z), \quad \omega^{(r)} \in[0,1], \quad z \in \mathbb{C} \backslash\{0\},
$$

where

$$
a(z)=-\frac{z^{-3}(z+1)^{4}}{16}\left(z^{2}-4 z+1\right)
$$

is the symbol of the four point scheme and

$$
b(z)=\frac{z^{-5}(z+1)^{6}}{256}\left(3 z^{4}-18 z^{3}+38 z^{2}-18 z+3\right)
$$

is the symbol of the $C^{2}$-convergent quintic Dubuc-Deslauriers subdivision scheme [18]. By Floater and Muntingh [25], the Hölder exponent of the limits of $S_{\mathbf{b}}$ is $\alpha \approx 2.8301$. To determine the regularity of the level and parameter dependent scheme with the symbols in (3.7) we consider the matrix set

$$
\mathcal{T}_{0,1}=\left\{\left.A_{\varepsilon, 0}\right|_{V_{2}},\left.A_{\varepsilon, 1}\right|_{V_{2}}, \varepsilon \in\{0,1\}\right\}
$$

with the four matrices

$$
\begin{aligned}
& \left.A_{0, \omega}\right|_{V_{2}}=\frac{1}{256}\left(\begin{array}{ccccccc}
3-3 \omega & 0 & 0 & 0 & 0 & 0 & 0 \\
-7-9 \omega & -9+9 \omega & 3-3 \omega & 0 & 0 & 0 & 0 \\
45+3 \omega & 45+3 \omega & -7-9 \omega & -9+9 \omega & 3-3 \omega & 0 & 0 \\
-9+9 \omega & -7-9 \omega & 45+3 \omega & 45+3 \omega & -7-9 \omega & -9+9 \omega & 3-3 \omega \\
0 & 3-3 \omega & -9+9 \omega & -7-9 \omega & 45+3 \omega & 45+3 \omega & -7-9 \omega \\
0 & 0 & 0 & 3-3 \omega & -9+9 \omega & -7-9 \omega & 45+3 \omega \\
0 & 0 & 0 & 0 & 0 & 3-3 \omega & -9+9 \omega
\end{array}\right), \\
& \left.A_{1, \omega}\right|_{V_{2}}=\frac{1}{256}\left(\begin{array}{ccccccc}
-9+9 \omega & 3-3 \omega & 0 & 0 & 0 & 0 & 0 \\
45+3 \omega & -7-9 \omega & -9+9 \omega & 3-3 \omega 0 & 0 & 0 & \\
-7-9 \omega & 45+3 \omega & 45+3 \omega & -7-9 \omega & -9+9 \omega & 3-3 \omega & 0 \\
3-3 \omega & -9+9 \omega & -7-9 \omega & 45+3 \omega & 45+3 \omega & -7-9 \omega & -9+9 \omega \\
0 & 0 & 3-3 \omega & -9+9 \omega & -7-9 \omega & 45+3 \omega & 45+3 \omega \\
0 & 0 & 0 & 0 & 3-3 \omega & -9+9 \omega & -7-9 \omega \\
0 & 0 & 0 & 0 & 0 & 0 & 3-3 \omega
\end{array}\right)
\end{aligned}
$$


for $\omega \in\{0,1\}$. In this case, by Theorem 3.4, the regularity of the non-stationary scheme $\left\{S_{\mathbf{a}\left(\omega^{(r)}\right)}, r \in \mathbb{N}\right\}$ coincides with the regularity of the four point scheme. For $\omega \in\{t, 1\}, t>0$, the scheme $\left\{S_{\mathbf{a}\left(\omega^{(r)}\right)}, r \in \mathbb{N}\right\}$ is $C^{2}$-convergent. Remarkably, for $\omega \in\{0, t\}$, $t<1$, extensive numerical experiments show that the JSR of the family $\mathcal{T}_{0, t}$ is determined by the the subfamily $\left\{\left.A_{\varepsilon, t}\right|_{V_{2}}, \varepsilon \in\{0,1\}\right\}$. For example, for $t=\frac{1}{2}$, we obtain $\rho\left(\mathcal{T}_{0, \frac{1}{2}}\right) \approx 0.2078$ and, thus, the corresponding Hölder exponent is $\alpha \geq 2.2662$.

The exact computations of the joint spectral radius in the above examples are done using the algorithms in [26,27].

\section{Acknowledgments}

Vladimir Protasov was sponsored by RFBR Grants 13-01-00642, 14-01-00332 and by the Grant of Dynasty foundation.

\section{References}

[1] C. Beccari, G. Casciola, L. Romani, A non-stationary uniform tension controlled interpolating 4-point scheme reproducing conics, Comput. Aided Geom. Des. 24 (2007) 1-9.

[2] D. Burkhart, B. Hamann, G. Umlauf, Iso-geometric finite element analysis based on Catmull-Clark subdivision solids, Comput. Graph. Forum 29 (2010) $1575-1584$

[3] C.A. Cabrelli, C. Heil, U.M. Molter, Self-similarity and multiwavelets in higher dimensions, Mem. Am. Math. Soc. 170 (807) (2004) 1-82.

[4] A.S. Cavaretta, W. Dahmen, C.A. Micchelli, Stationary subdivision, Mem. Am. Math. Soc. 453 (i-vi) (1991) 1-185.

[5] M. Charina, Vector multivariate subdivision schemes: comparison of spectral methods for their regularity analysis, Appl. Comput. Harmon. Anal. 32 (2012) 86-108.

[6] M. Charina, C. Conti, K. Jetter, G. Zimmermann, Scalar multivariate subdivision schemes and box splines, Comput. Aided Geom. Des. 28 (2011) 285-306.

[7] M. Charina, C. Conti, N. Guglielmi, V. Protasov, Regularity of non-stationary subdivision: a matrix approach (submitted for publication) (arXiv:1406.7131).

[8] D.R. Chen, R.Q. Jia, S.D. Riemenschneider, Convergence of vector subdivision schemes in Sobolev spaces, Appl. Comput. Harmon. Anal. 12 (2002) 128-149.

[9] F. Cirak, M. Ortiz, P. Schröder, Subdivision surfaces: a new paradigm for thin-shell finite-element analysis, Int. J. Numer. Methods Eng. 47 (2000) $2039-2072$.

[10] A. Cohen, N. Dyn, Nonstationary subdivision schemes and multiresolution analysis, SIAM J. Math. Anal. 27 (1996) $1745-1769$.

[11] C. Conti, Stationary and nonstationary affine combination of subdivision masks, Math. Comput. Simul. 81 (2010) 623-635.

[12] C. Conti, L. Gori, F. Pitolli, Totally positive functions through nonstationary subdivision schemes, J. Comput. Appl. Math. 200 (2007) 255-265.

[13] C. Conti, L. Gori, F. Pitolli, P. Sablonniere, Approximation by GP box-splines on a four-direction mesh, J. Comput. Appl. Math. 221 (2008) 310-329.

[14] C. Conti, L. Romani, Affine combination of B-splines subdivision masks and its non-stationary counterparts, BIT Numer. Math. 50 (2010) 269-299.

[15] W. Dahmen, C.A. Micchelli, Biorthogonal wavelet expansions, Constr. Approx. 13 (1997) 293-328.

[16] I. Daubechies, J.C. Lagarias, Sets of matrices all infinite products of which converge, Linear Algebra Appl. 162 (1992) $227-263$.

[17] R. Delgado-Gonzalo, P. Thevenaz, C.S. Seelamantula, M. Unser, Snakes with an ellipse reproducing property, IEEE Trans. Image Process. 21 (2012) 1258-1271.

[18] G. Deslauriers, S. Dubuc, Symmetric iterative interpolation processes, Constr. Approx. 5 (1989) 49-68.

[19] N. Dyn, J.A. Gregory, D. Levin, A four-point interpolatory subdivision scheme for curve design, Comput. Aided Geom. Des. 4 (1987) 257-268.

[20] N. Dyn, D. Levin, Analysis of asymptotically equivalent binary subdivision schemes, J. Math. Anal. Appl. 193 (1995) 594-621.

[21] N. Dyn, D. Levin, J.A. Gregory, A butterfly subdivision scheme for surface interpolation with tension control, ACM Trans. Graph. 9 (1990) 160-169.

[22] L. Elsner, The generalized spectral-radius theorem: an analytic-geometric proof, Linear Algebra Appl. 220 (1995) 151-159.

[23] M.-e. Fang, W. Ma, G. Wang, A generalized curve subdivision scheme of arbitrary order with a tension parameter, Comput. Aided Geom. Des. 27 (2010) $720-733$.

[24] M.-e. Fang, W. Ma, G. Wang, A generalized surface subdivision scheme of arbitrary order with a tension parameter, Comput. Aided Des. 49 (2014) 8-17.

[25] M.S. Floater, G. Muntingh, Exact regularity of pseudo-splines, arXiv:1209.2692.

[26] N. Guglielmi, V. Protasov, Exact computation of joint spectral characteristics of matrices, Found. Comput. Math. 13 (2013) $37-97$.

[27] N. Guglielmi, F. Wirth, M. Zennaro, Complex polytope extremality results for families of matrices, SIAM J. Matrix Anal. Appl. 27 (2005) $721-743$.

[28] N. Guglielmi, M. Zennaro, On the asymptotic properties of a family of matrices, Linear Algebra Appl. 322 (2001) 169-192.

[29] B. Han, Vector cascade algorithms and refinable function vectors in Sobolev spaces, J. Approx. Theory 124 (2003) 44-88.

[30] K. Jetter, G. Plonka, A survey on $L_{2}$-Approximation order from shift-invariant spaces, in: N. Dyn, D. Leviatan, D. Levin, A. Pinkus (Eds.), Multivariate Approximation and Applications, Cambridge University Press, 2001, pp. 73-111.

[31] R.-Q. Jia, Subdivision schemes in $l_{p}$ spaces, Adv. Comput. Math. 3 (1995) 309-341.

[32] D. Levin, Using Laurent polynomial representation for the analysis of non-uniform binary subdivision schemes, Adv. Comput. Math. 11 (1999) 41-54.

[33] C. Möller, U. Reif, A tree-based approach to joint spectral radius determination, Linear Algebra Appl. 563 (2014) 154-170.

[34] J. Peter, U. Reif, Subdivision Surfaces, Geometry and Computing, vol. 3, Springer-Verlag, Berlin, 2008.

[35] G.-C. Rota, G. Strang, A note on the joint spectral radius, Indag. Math. 22 (1960) 379-381.

[36] V. Uhlmann, R. Delgado-Gonzalo, C. Conti, L. Romani, M. Unser, Exponential Hermite splines for the analysis of biomedical images, in: Proceedings of the IEEE International Conference on Acoustic, Speech and Signal Processing (ICASSP), 2014, pp. 1650-1653.

[37] J. Warren, H. Weimer, Subdivision Methods for Geometric Design, Morgan-Kaufmann, 2002. 